PAPER • OPEN ACCESS

Electron beam induced synthesis of Ru-rGO and its super capacitive behavior

To cite this article: Nilesh G Saykar et al 2019 2D Mater. 6045030

View the article online for updates and enhancements. 


\title{
2D Materials
}

\section{OPEN ACCESS}

CrossMark

\section{RECEIVED}

3 October 2018

REVISED

4 July 2019

ACCEPTED FOR PUBLICATION

17 July 2019

PUBLISHED

16 August 2019

Original content from

this work may be used

under the terms of the

Creative Commons

Attribution 3.0 licence.

Any further distribution

of this work must

maintain attribution

to the author(s) and the

title of the work, journal

citation and DOI.

\section{PAPER}

\section{Electron beam induced synthesis of Ru-rGO and its super capacitive behavior}

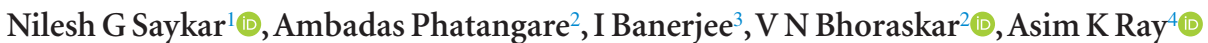 \\ and $S \mathrm{~K}$ Mahapatra ${ }^{1,5}$ \\ 1 Department of Physical Sciences, Central University of Punjab, Bathinda, Punjab, India \\ 2 Department of Physics, University of Pune, Ganeshkhind, Pune, India \\ 3 School of Nano Sciences, Central University of Gujarat, Gandhinagar, Gujarat, India \\ 4 Department of Electronic and Computer Engineering, Brunel University London, Uxbridge, Middlesex, UB8 3PH, United Kingdom \\ Author to whom any correspondence should be addressed. \\ E-mail: skmahapatra741973@gmail.com
}

Keywords: electron beam irradiation, supercapacitor, Ru-rGO

\begin{abstract}
We report an in situ synthesis of ruthenium-reduced graphene oxide (Ru-rGO) using $6 \mathrm{MeV}$ electron beam assisted radiolytic reduction method and its supercapacitive behavior. X-ray diffraction (XRD), transmission electron microscopy (TEM) and field emission scanning electron microscopy (FESEM) explore Ru nanoparticles of size $\sim 2 \mathrm{~nm}$ are decorated on rGO sheets. Raman spectroscopy shows $I_{\mathrm{D}} / I_{\mathrm{G}}$ ratio increased and formation of bilayer $\mathrm{rGO}$ after electron beam irradiation. The defect density in Ru-rGO is increased due to the electron beam irradiation as compared to its counterpart GO. The Ru-rGO based supercapacitor exhibits specific capacitance (128.1 \pm 5.59$) \mathrm{F} \mathrm{g}^{-1}$ at $10 \mathrm{mV}$ $\mathrm{s}^{-1}$ scan rate. The specific capacitance retention of Ru-rGO is up to $99.4 \%$ at 900 cycles while it increases to $130 \%$ at 5000 cycles. Discharge curve of the supercapacitor involves three current decay processes viz. activation polarization, ohmic polarization and concentration polarization. The highest energy density of $(4.125 \pm 0.19) \mathrm{W} \mathrm{h} \mathrm{kg}^{-1}$ and power density of $1.44 \mathrm{~kW} \mathrm{~kg}^{-1}$ are achieved with Ru-rGO supercapacitor. This unique electron beam assisted techniques illustrates a promising method of the fabrication of high performance supercapacitor.
\end{abstract}

\section{Introduction}

High-performance portable and fast response energy storage devices (ESDs) are vital for future needs [1-5]. Two dimensional (2D) materials have the potential to fulfil these energy needs on the basis of their electrical and chemical properties [6-8]. 2D materials such as graphene, graphene derivatives, transition metal dichalcogenides, transition metal carbides/nitrides (MXene), and 2D metal-organic frameworks (MOFs) are being used in electrochemical ESDs [9-11]. Graphene and its derivatives could be efficient material for ESDs as they have excellent electrical and chemical properties as well as exceptionally high surface area [12-14]. The theoretical capacitance value of $\sim 550 \mathrm{~F}$ $\mathrm{g}^{-1}$ is reported for graphene [15]. rGO is one of the graphene derivative, well-known in electrochemical supercapacitors/ultracapacitors due to its unique properties of rapid charging/discharging [14, 16], and storing energy for long times [17]. Hence, a high level of research interest is oriented towards graphene and its derivatives (especially $\mathrm{rGO}$ ) in order to explore their electrochemical properties [18-20]. Aggregation and restacking of graphene sheets reduce the intrinsic surface area which lower the capacitance value [21]. Literature revels that intercalation of polymers, nanoparticles, and carbon nanotubes could avoid the restacking of graphene sheets [22-24]. In this view, graphene and its derivatives have been combined with metal and metal oxide nanoparticles for the enhancement of electrochemical properties [25-28]. $\mathrm{RuO}_{2}-\mathrm{rGO}$ composite is widely reported as the best electrode material for energy storage [26, 27, 29].

Number of layers, wrinkles, defects and functional group of graphene and its derivatives have a huge impact of the synthesis method, which affect the specific surface area and defect density. Therefore, an appropriate physical or chemical method is essential to obtain desirable graphene-based materials. Conventionally, metal nanoparticle assembled with rGO $\left(m_{\mathrm{np}}-\mathrm{rGO}\right)$ is prepared through ex-situ and in situ chemical method [30]. In the case of the ex-situ 
process, the metal nanoparticles are synthesized separately and then mixed with the precursor of GO to produce $m_{\mathrm{np}}$-rGO which results in less loading of nanoparticles [31]. In the case of in situ method, the precursor of metal and GO are mixed to produce $m_{\mathrm{np}}$ rGO but unstable $m_{\mathrm{np}}$-rGO are formed due to the removal of oxygen-containing groups [32]. Researchers are trying to synthesize $m_{\mathrm{np}}$-rGO through different synthesis methods such as green synthesis [33], hydrothermal method [34] and electrochemical method [35] to improve the stability and loading capability. Shayeh et al synthesized Polyaniline/reduced graphene oxide/Au composite by electrochemical deposition method and prepared hybrid supercapacitor which exhibits specific capacitance $303 \mathrm{~F} \mathrm{~g}^{-1}$ at $1 \mathrm{~mA} \mathrm{~cm}{ }^{-2}$ [36]. The graphene-platinum nanoparticles prepared through chemically reduced exfoliated method shows a significantly larger capacitance of $269 \mathrm{~F} \mathrm{~g}^{-1}$ at a scan rate of $50 \mathrm{mV} \mathrm{s}^{-1}$ [37]. Ru-rGO synthesized using microwave method, delivers a maximum specific capacitance of $140 \mathrm{~F} \mathrm{~g}^{-1}$ at $10 \mathrm{mV} \mathrm{s}^{-1}$, and maximum energy and power densities of $8.4 \mathrm{~W} \mathrm{~h} \mathrm{~kg}^{-1}$ and 7.8 $\mathrm{kW} \mathrm{kg}^{-1}$, respectively [38]. CNT/graphene/porous nickel prepared by chemical vapour deposition exhibits specific capacitance of $100 \mathrm{~F} \mathrm{~g}^{-1}$ at $1 \mathrm{~A} \mathrm{~g}^{-1}$ [39]. A comparison of the electrochemical performance of $2 \mathrm{D}$ electrode materials for supercapacitor devices is shown in table 1.

A few reports are available on the electron beam assisted preparation of $m_{\mathrm{np}}-\mathrm{rGO}$ [40]. The basic advantage of the electron beam induced method is hydrated electrons with high negative redox potential act as a reducing agent which enables any metal ions to be reduced to their zero valent metal atoms. The process undergoes homogeneous nucleation with the nucleation sites being generated by the primary atoms at the original sites independently like the precursor ions. The process leads to uniformly dispersed and highly stable nanoparticles without the involvement of any chemical reducing agent. Furthermore, the important properties of graphene-based materials like defects, number of layers and size of nanoparticles can be tuned through the imposed dose of the electron [41]. In this view, a simple in situ electron beam assisted reduction method for preparation of $m_{\mathrm{np}}$ rGO is gained importance to fabricate stable and loading tunable $m_{\mathrm{np}}$-rGO.

The present work focusses on the in situ synthesis of Ru-rGO using a $6 \mathrm{MeV}$ electron beam assisted reduction method and the study of its supercapacitive behavior. The structural and morphological properties of GO and Ru-rGO are studied using XRD, Raman spectroscopy, FESEM and TEM. Electrochemical properties like cyclic voltammetry $(\mathrm{CV})$, galvanostatic charging and discharging are studied. The developed Ru-rGO based super capacitor exhibits specific capacitance of $(128 \pm 5.59) \mathrm{F} \mathrm{g}^{-1}$ with $10 \mathrm{mV} \mathrm{s}^{-1}$ scan rate and specific capacitance retention up to $99.4 \%$ at 900 cycles while it increases to $130 \%$ at 5000 cycles. The results obtained with this unique beam assisted technique may be useful for the fabrication of high-performance supercapacitor based on Ru-rGO.

\section{The mechanism for nucleation and growth of Ru-rGO}

The electron beam assisted a radiolytic reduction is a strong tool for synthesizing monodispersed metallic nanoparticles. The process here has been used to decorate 1D rGO sheets with highly dispersed nanoparticles of ruthenium. The radiolytic reduction process in aqueous medium involves irradiation of $\mathrm{H}_{2} \mathrm{O}$ to produce active radicals of hydrated electrons $\left(e_{\mathrm{aq}}^{-}\right), \mathrm{H} \cdot$ and $\cdot \mathrm{OH}$.

$$
\mathrm{H}_{2} \mathrm{O}+\text { e beam } \rightarrow e^{-}, \mathrm{H}^{*}, \cdot \mathrm{OH} .
$$

Where, $\cdot \mathrm{OH}$ and $\mathrm{H} \cdot$ are the hydroxyl and hydrogen radicals respectively. These radicals are strong reducing agents having high redox potentials of $E^{0}$ $\left(\mathrm{H}_{2} \mathrm{O} / e_{\mathrm{aq}}^{-}\right)=-2.87 V_{\mathrm{NHE}}, E^{0}\left(\mathrm{H}^{+} / \mathrm{H} \cdot\right)=-2.3 V_{\mathrm{NHE}}$, $E^{0} \quad\left(\mathrm{OH} \cdot / \mathrm{H}_{2} \mathrm{O}\right)=+2.8 \quad V_{\text {NHE }}$ etc. These radicals reduce metal ions into a zero valent state. When $\mathrm{RuCl}_{3}$ is dissolved in $\mathrm{H}_{2} \mathrm{O}$ and irradiated with an e beam, hydrated $e^{-}$reacts with $\mathrm{Ru}(\mathrm{III}) \mathrm{Cl}_{3}^{-}$to produce stable Ru nanoparticles [51].

$$
n \mathrm{Ru}^{3+}+n e^{-} \rightarrow n \mathrm{Ru}^{0} .
$$

To prevent the oxidation process, $\mathrm{OH}$. scavengers like isopropyl alcohol (IPA) are used in the aqueous medium of the precursor solution to form reducing species [52].

$$
\begin{aligned}
& \left(\mathrm{CH}_{3}\right)_{2} \mathrm{CHOH}+\cdot \mathrm{OH} \rightarrow \mathrm{H}_{2} \mathrm{O}+\left(\mathrm{CH}_{3}\right)_{2} \mathrm{C} \cdot \mathrm{OH} / \cdot \\
& \mathrm{CH}_{2} \mathrm{CH}\left(\mathrm{CH}_{3}\right) \mathrm{OH} /\left(\mathrm{CH}_{3}\right)_{2} \mathrm{CHO} \cdot .
\end{aligned}
$$

When GO and $\mathrm{RuCl}_{3}$ are dispersed in IPA and irradiated with e-beam, the following reaction takes place [53].

$$
\begin{aligned}
& n\left[\left(\mathrm{CH}_{3}\right)_{2} \mathrm{C} \cdot(\mathrm{OH})\right]+\mathrm{GO} \rightarrow n\left[\left(\mathrm{CH}_{3}\right)_{2} \mathrm{CO}\right]+\operatorname{Red~GO}(\mathrm{H}) \\
& n \mathrm{Ru}^{3+}+n\left[\left(\mathrm{CH}_{3}\right)_{2} \mathrm{C} \cdot(\mathrm{OH})\right] \rightarrow n \mathrm{Ru}^{0} \\
& +n\left[\left(\mathrm{CH}_{3}\right)_{2} \mathrm{CO}\right]+n \mathrm{H}^{+}
\end{aligned}
$$

The overall reaction of $\mathrm{GO}$ and $\mathrm{Ru}$ reduction, therefore, follows as

$$
\begin{aligned}
& n\left[\left(\mathrm{CH}_{3}\right)_{2} \mathrm{C} \cdot(\mathrm{OH})\right]+\mathrm{GO}+n \mathrm{Ru}^{3+}+n e^{-} \\
& \rightarrow \operatorname{Red~GO}(H)+n \mathrm{Ru}^{0}+n\left[\left(\mathrm{CH}_{3}\right)_{2} \mathrm{CO}\right]+n \mathrm{H}^{+} .
\end{aligned}
$$

The reducing agent generated by the radiation can penetrate deep into the sample solution causing random reduction all through the sample volume. The $\mathrm{Ru}$ atoms formed now act as individual sites for nucleation further followed by coalescence. The atomatom and atom with unreduced atoms have binding energies much higher than atom-solvent or atomligand [51]. This causes atom-atom combination. When large numbers of atoms are generated through radiolysis cluster formation is initiated. The charged 
Table 1. Comparison of electrochemical performance of 2D material electrodes for supercapacitor devices.

\begin{tabular}{|c|c|c|c|c|c|}
\hline Material & Synthesis method & Specific capacitance & $\begin{array}{l}\text { Specific capacitance } \\
\text { retention }\end{array}$ & $\begin{array}{l}\text { Energy density } \\
@ \text { power density } \\
\left(\mathrm{Wh} \mathrm{kg}{ }^{-1} @ \mathrm{~kW}\right. \\
\left.\mathrm{kg}^{-1}\right)\end{array}$ & Ref. \\
\hline rGO-AuNPs & Electrochemical reduction & $357 \mathrm{~F} \mathrm{~g}^{-1}\left(25 \mathrm{mv} \mathrm{s}^{-1}\right)$ & $75 \%$ (1000 cycles) & - & {$[42]$} \\
\hline Ag-rGO & Ultrasonication/reduction & $220 \mathrm{~F} \mathrm{~g}^{-1}\left(10 \mathrm{mV} \mathrm{s}^{-1}\right)$ & - & - & {$[28]$} \\
\hline $\mathrm{Au} / \mathrm{ZnO} / \mathrm{rGO}$ & $\begin{array}{l}\text { Microwave-assisted } \\
\text { hydrothermal }\end{array}$ & $875 \mathrm{Fg}^{-1}\left(1 \mathrm{~A} \mathrm{~g}^{-1}\right)$ & $62 \%(1000$ cycles $)$ & $36.5 @ 0.27$ & {$[43]$} \\
\hline $\mathrm{Ni} / \mathrm{NiO}-\mathrm{rGO}$ & Probe sonication & $310 \mathrm{Cg}^{-1}\left(1 \mathrm{~A} \mathrm{~g}^{-1}\right)$ & 95\% (1000 cycles) & $14.6 @ 4.3$ & {$[44]$} \\
\hline rGO- $\mathrm{MoS}_{2}$ & Hydrothermal & $318 \mathrm{Fg}^{-1}\left(1 \mathrm{mV} \mathrm{s}^{-1}\right)$ & $82 \%$ (5000 cycles) & $44.1 @ 0.159$ & {$[45]$} \\
\hline rGO-MoS 2 & Hydrothermal & $387.6 \mathrm{~F} \mathrm{~g}^{-1}\left(1.2 \mathrm{~A} \mathrm{~g}^{-1}\right)$ & 100\% (1000 cycles) & 220@4.0 & {$[46]$} \\
\hline $\mathrm{MoSe}_{2}$-graphene & $\begin{array}{l}\text { Sonochemical assisted } \\
\text { exfoliation }\end{array}$ & $945 \mathrm{~F} \mathrm{~g}^{-1}\left(1 \mathrm{~A} \mathrm{~g}^{-1}\right)$ & $92 \%$ (3000 cycles) & $26.6 @ 0.8$ & {$[47]$} \\
\hline $\mathrm{rGO}-\mathrm{SnO}_{2}$ & Sol-gel/thermal reduction & $229.1 \mathrm{~F} \mathrm{~g}^{-1}\left(2 \mathrm{mv} \mathrm{s}^{-1}\right)$ & $82 \%$ (1000 cycles) & - & {$[48]$} \\
\hline $\mathrm{rGO}-\mathrm{RuO}_{2}$ & Sol gel & $500 \mathrm{~F} \mathrm{~g}^{-1}\left(1.0 \mathrm{~A} \mathrm{~g}^{-1}\right)$ & $87 \%$ (2000 cycles) & $16.7 @ 1.0$ & {$[29]$} \\
\hline $\mathrm{rGO}-\mathrm{Ti}_{3} \mathrm{C}_{2} \mathrm{Tx}$ & Etching and sonication & $254 \mathrm{Fg}^{-1}\left(\mathrm{mV} \mathrm{s}^{-1}\right)$ & - & - & {$[49]$} \\
\hline $\mathrm{V}_{4} \mathrm{C}_{3}$ & Etching & $209 \mathrm{~F} \mathrm{~g}^{-1}\left(2 \mathrm{mV} \mathrm{s}^{-1}\right)$ & $97.23 \%$ (10 000 cycles) & - & {$[50]$} \\
\hline Ru-rGO & Electron beam irradiation & $128 \mathrm{~F} \mathrm{~g}^{-1}\left(10 \mathrm{mV} \mathrm{s}^{-1}\right.$ & $\begin{array}{l}99.4 \% \text { up to } 500 \text { cycles } \\
(130 \% 5000 \text { cycles })\end{array}$ & $4.1 @ 0.6$ & This work \\
\hline
\end{tabular}

dimer clusters undergo further reduction to form more nucleation sites. The rate of reduction of free metal ions and cluster formation is controlled by the rate of reducing agent formation. The bonding between clusters with unreduced ions or two charged clusters is strong enough for growth to take place. The final cluster size depends on the limitation applied to the coalescence process beyond a certain nuclear size formation.

\section{Experimental details}

\subsection{In situ synthesis of Ru-rGO by electron beam irradiation}

GO was synthesized by a modified Hummers' method, the preparation procedure is explained elsewhere $[40,54]$. The prepared GO sample is dispersed in IPA $\left(0.5 \mathrm{mg} \mathrm{ml}^{-1}\right)$ and sonicated for $1 \mathrm{~h} .25 \mathrm{mM}$ of $\mathrm{RuCl}_{3}$ is dissolved in double distilled water and stirred for $30 \mathrm{~min}$. $2 \mathrm{ml}$ of $\mathrm{GO}$ dispersion and $\mathrm{RuCl}_{3}$ solution is taken in a thin plastic bottle and exposed to a $6 \mathrm{MeV}$ electron beam obtained from a Racetrack Microtron Accelerator having pulse width of $2 \mu$ s and a pulse repetition rate of 50 pulse $\mathrm{s}^{-1}$. The sample is exposed to $6 \mathrm{MeV}$ electron beam of fluency $1 \times 10^{15} e^{-} \mathrm{cm}^{-2}$. After irradiation, the sample is centrifuged, washed with double distilled water and dried. A powdered sample is used for XRD using a Bruker AXS D8 Advance X-ray diffractometer with $\mathrm{Cu}_{\mathrm{K} \alpha}$ radiation at a wavelength of $1.5406 \AA$. The surface morphology of GO and Ru-rGO are obtained by preparing samples using a drop cast method on a silicon wafer and using a FESEM (Carl Zeiss Merlin Compact). Raman spectra are obtained using a Renishaw Invia laser Raman microscope with a laser excitation wavelength of $532 \mathrm{~nm}$. Particle size and crystal structure of samples are obtained using TEM (TECNAI g2).

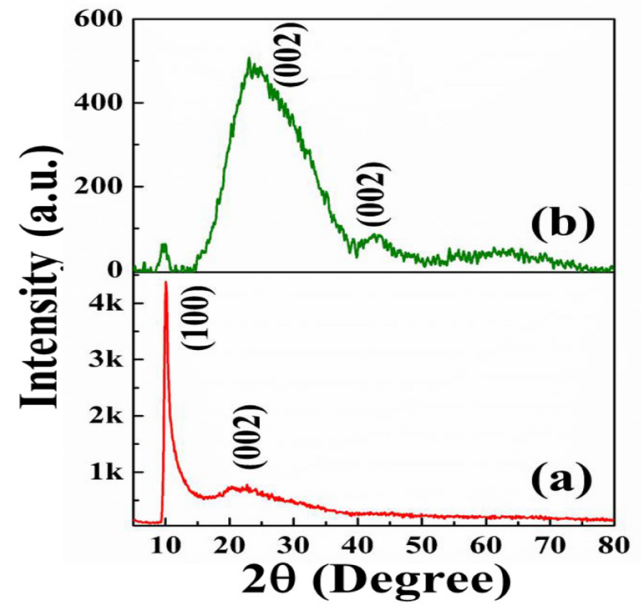

Figure 1. XRD spectra of (a) GO and (b) Ru-rGO.

\subsection{Electrochemical measurement}

Electrochemical measurements are performed using a $\mathrm{CHI} 760 \mathrm{E}$ electrochemical workstation (CHI instruments, USA). A PEDOT is used as a binder for the electrode preparation. Both samples of GO and $\mathrm{Ru}-\mathrm{rGO}$ were prepared individually mixed with PEDOT in 8:2 weight ratio and stirred continuously to obtain a homogeneous slurry. Glassy carbon with diameter $3 \mathrm{~mm}$ is used as a working electrode while $\mathrm{Ag} / \mathrm{AgCl}$ and platinum wire are used as reference and counter electrode respectively. The prepared slurry is cast on the glassy carbon electrode. The mass loading on the electrode is about $2 \mathrm{mg}$ and is kept constant for all samples. The mass of the active material GO and Ru-rGO is measured by weighing the electrode before and after rGO deposition using a high precision laboratory balance. Studies related to $\mathrm{CV}$ and charging-discharging of $\mathrm{GO}$ and $\mathrm{Ru}-\mathrm{rGO}$ 




Figure 2. FE-SEM images of (a) GO (b) Ru-rGO and EDS spectra of (c) GO, (d) Ru-rGO (inset shows elemental weight\% and atomic\%).

materials are performed using a three-electrode cell configuration in $1 \mathrm{M} \mathrm{H}_{2} \mathrm{SO}_{4}$ electrolyte, within a potential window of 0 to $0.9 \mathrm{~V}$ at different scan rate ranging from 10 to $500 \mathrm{mV} \mathrm{s}^{-1}$. The reproducibility of the electrochemical measurement results has been examined by performing measurements on four samples of each GO and Ru-rGO.

\section{Results and discussion}

XRD spectra of GO and Ru-rGO are shown in figures 1(a) and (b). Figure 1(a) shows a strong diffraction peak at $10.07^{\circ}$ corresponds to the $(100)$ plane of GO and another broad peak around $22.68^{\circ}$ corresponding to $(002)$ is due to the partially restacked graphite structure [55]. After electron irradiation, it is seen (figure $1(\mathrm{~b})$ ) that new peaks at $25.76^{\circ}$ correspond to the $(002)$ plane of $\mathrm{rGO}$ and $42.59^{\circ}$ correspond to (002) plane of ruthenium [JCPDS-ICDD 06-0663] are observed [26]. Crystallite size and interplanar distance of ruthenium are $2 \mathrm{~nm}$ and $0.2381 \mathrm{~nm}$ calculated through the Debye Scherer formula and Bragg's law respectively. Furthermore, it is found that the average crystallite size $\left(D_{002}\right)$ and the interplanar distance $\left(d_{002}\right)$ of rGO $(002)$ plane are $0.62 \mathrm{~nm}$ and $0.343 \mathrm{~nm}$ respectively. The number of $\mathrm{rGO}$ layers $(N)$ is calculated using the expression $D_{002}=(N-1)$ $d_{002}$, where $D_{002}$ is assumed to represent the thickness as measured from the centre of the sample [56]. It shows that rGO has two (2.81) layers. Electron beam irradiation has simultaneously reduced GO to rGO and $\mathrm{Ru}^{3+}$ to $\mathrm{Ru}^{0}$. XRD spectra confirm the presence of $\mathrm{Ru}$ on $\mathrm{rGO}$ sheets and/or intercalate between the rGO sheets.

The FESEM images of GO and Ru-rGO are shown in figure 2. Figure 2(a) shows wrinkle sheet of GO whereas figure 2(b) shows well-separated sheets of rGO with the presence of ruthenium nanoparticles on it. In addition, ruthenium nanoparticles are seen underneath rGO sheets like spacers, which is in accordance with the XRD and Raman results. Figures 2(c) and (d) show the EDS spectra of GO and Ru-rGO respectively. Figure 2(d) shows a peak of Ru in Ru-rGO samples whereas the peak corresponding to $\mathrm{Ru}$ seems to be absent in figure 2(c) in the GO sample. The RurGO sample shows a lower atomic oxygen percentage $(65.35 \%)$ than that of GO (inset of figures 2(c) and (d)). EDS spectra support the reduction of GO and Ru incorporated in $\mathrm{rGO}$ after electron beam irradiation.

TEM images of GO and Ru-rGO are shown in figure 3. They show a uniform distribution of Ru nanoparticles on rGO sheets. TEM images also illustrate the formation of a few layers of Ru-rGO sample. The inset in figure 3(b) shows atomic planes corresponding to $\mathrm{Ru}$ atoms. The presence of $\mathrm{Ru}$ nanoparticles has been confirmed from the selective area electron diffraction (SAED) pattern which confirms the polycrystalline nature of the Ru-rGO sample. The interplanar distance 

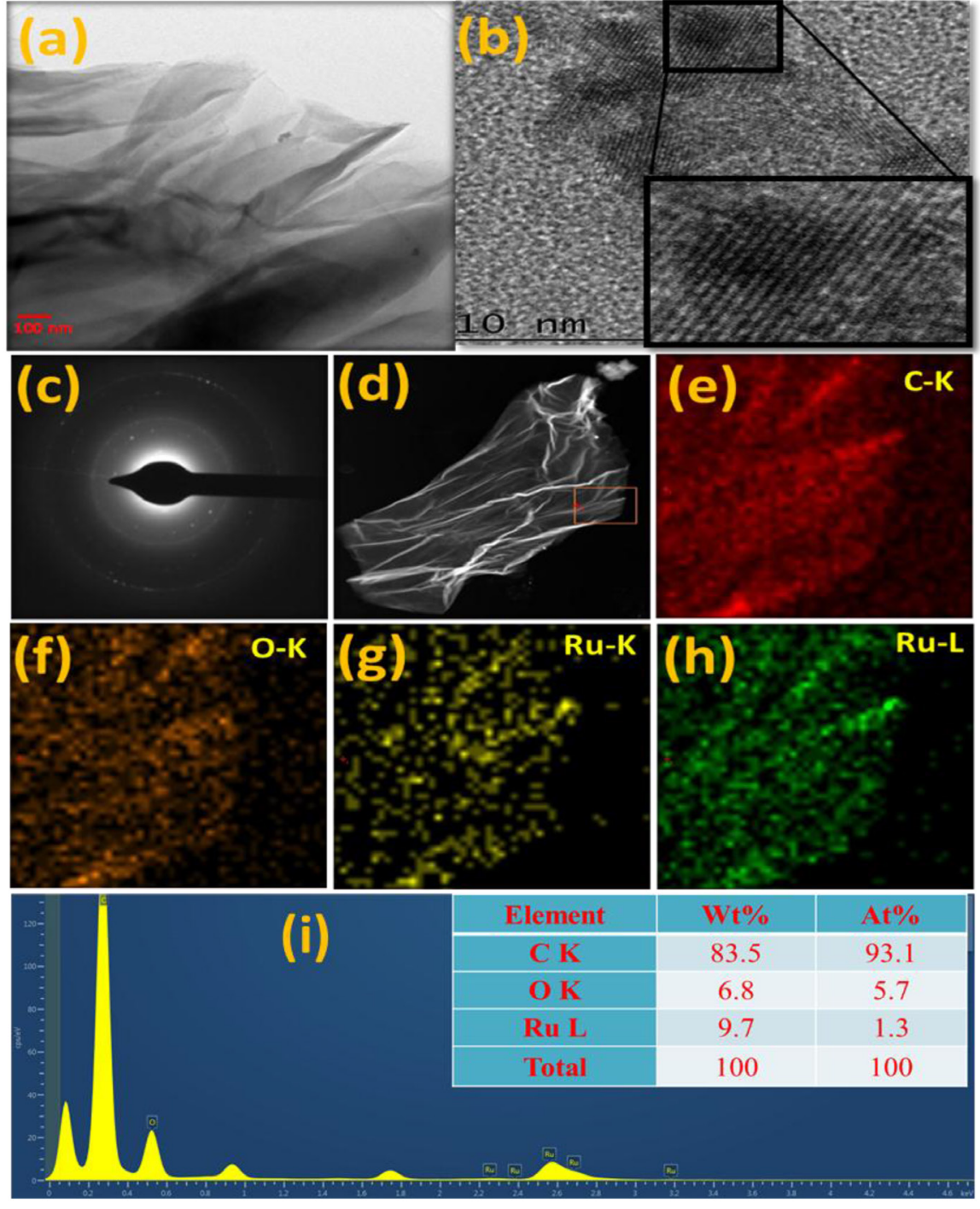

(i)
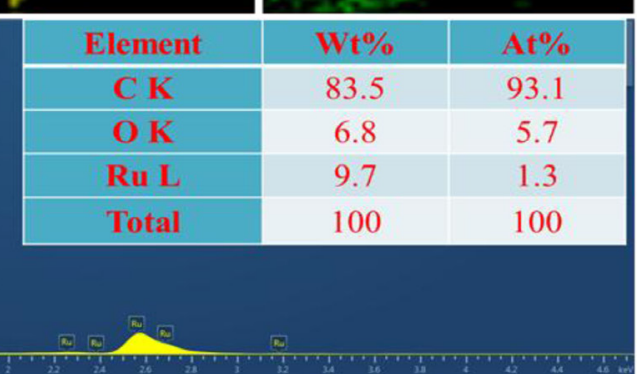

Figure 3. TEM of (a) GO, (b) Ru-rGO, inset atomic planes of Ru (c) SAED pattern of Ru (d) elemental mapping of Ru-rGO (e) C in $\mathrm{Ru}-\mathrm{rGO}$, (f) O in Ru-rGO, (g) Ru-K in Ru-rGO and (h) Ru-L in Ru-rGO (i) EDS spectra of Ru-rGO (inset shows elemental weight\% and atomic\% in Ru-rGO).

of the lattice for $\mathrm{Ru}$ is calculated to be $0.2341 \mathrm{~nm}$ and $0.1234 \mathrm{~nm}$ corresponding to $(002)$ and (103) planes respectively and it is an excellent agreement with the XRD data for the (002) plane. Elemental mapping is shown in figures 3(d)-(h) Elemental mapping reveals the homogeneous distribution of $\mathrm{Ru}$ nanoparticles on rGO sheets. EDS spectra of Ru-rGO (figure 3(i)) a characteristic peaks of $\mathrm{C}, \mathrm{O}$ and $\mathrm{Ru}$ which confirms the coexistence of these elements. An atomic percentage of C, $\mathrm{O}$ and Ru element in Ru-rGO is about 93.1\%, 5.7\% and $1.3 \%$ respectively (inset of figure $3(\mathrm{i})$ ).

The Raman spectra of GO and Ru-rGO are shown in figures $4(\mathrm{a})$ and (b). Figure $1(\mathrm{a})$ shows characteristic peaks of GO at around $1341 \mathrm{~cm}^{-1}$ and $1591 \mathrm{~cm}^{-1}$ corresponds to the vibrational bands of carbon in disordered $\mathrm{D}$ band and the $E_{2 \mathrm{~g}}$ mode of the graphitic $\mathrm{G}$ band [57-59]. After electron beam irradiation, it is seen that the red shift of the D peak attributed to the presence of $\mathrm{Ru}$ nanoparticles on/between the rGO sheets. However, no signature of formation of metal oxides like
$\mathrm{Ru}-\mathrm{O}$ and $\mathrm{RuO}_{2}$ which are expected to occur around $200-800 \mathrm{~cm}^{-1}$ [60]. This clearly indicates a direct reduction of $\mathrm{Ru}$ nanoparticles without the formation of any oxygenated ruthenium species. The electron irradiation mechanism seems to be strong enough to cause a direct reduction of $\mathrm{Ru}^{3+}$ ions into $\mathrm{Ru}^{0}$ nano particles with simultaneous binding to the GO sheets having high electron charge density at the surface. The D to $G$ band intensity ratio $\left(I_{\mathrm{D}} / I_{G}\right)$ is used to assess the crystalline structure of the synthesized material. The increase in the $I_{\mathrm{D}} / I_{G}$ from 0.84 to 0.90 shows an enhanced degree of graphitization after electron beam irradiation.

The Raman spectrum of Ru-rGO shows an additional peak around $2657 \mathrm{~cm}^{-1}$ attributed to the $2 \mathrm{D}$ band. The peak at $2937 \mathrm{~cm}^{-1}$ corresponding to D + D' band appears due to the combination of phonons with different momenta, around $K$ and $\Gamma$ points [61]. After electron beam irradiation, defects are induced in the Ru-rGO sample activating the $\mathrm{D}+\mathrm{D}^{\prime}$ band in the Raman spectrum observed at $2937 \mathrm{~cm}^{-1}$ [61]. The 


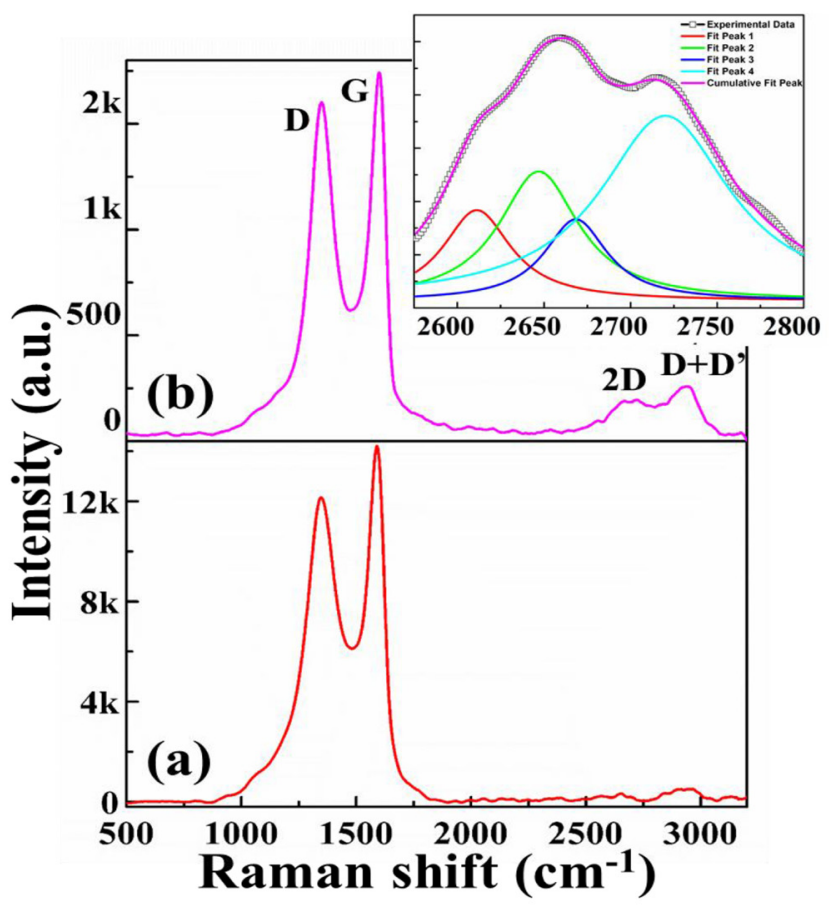

Figure 4. Raman spectra of (a) GO, (b) Ru-rGO inset of (b) shows peak fitting with the Lorentzian function of Raman spectra of $\mathrm{Ru}-\mathrm{rGO}$ in the range of 2575 to $2800 \mathrm{~cm}^{-1}$.
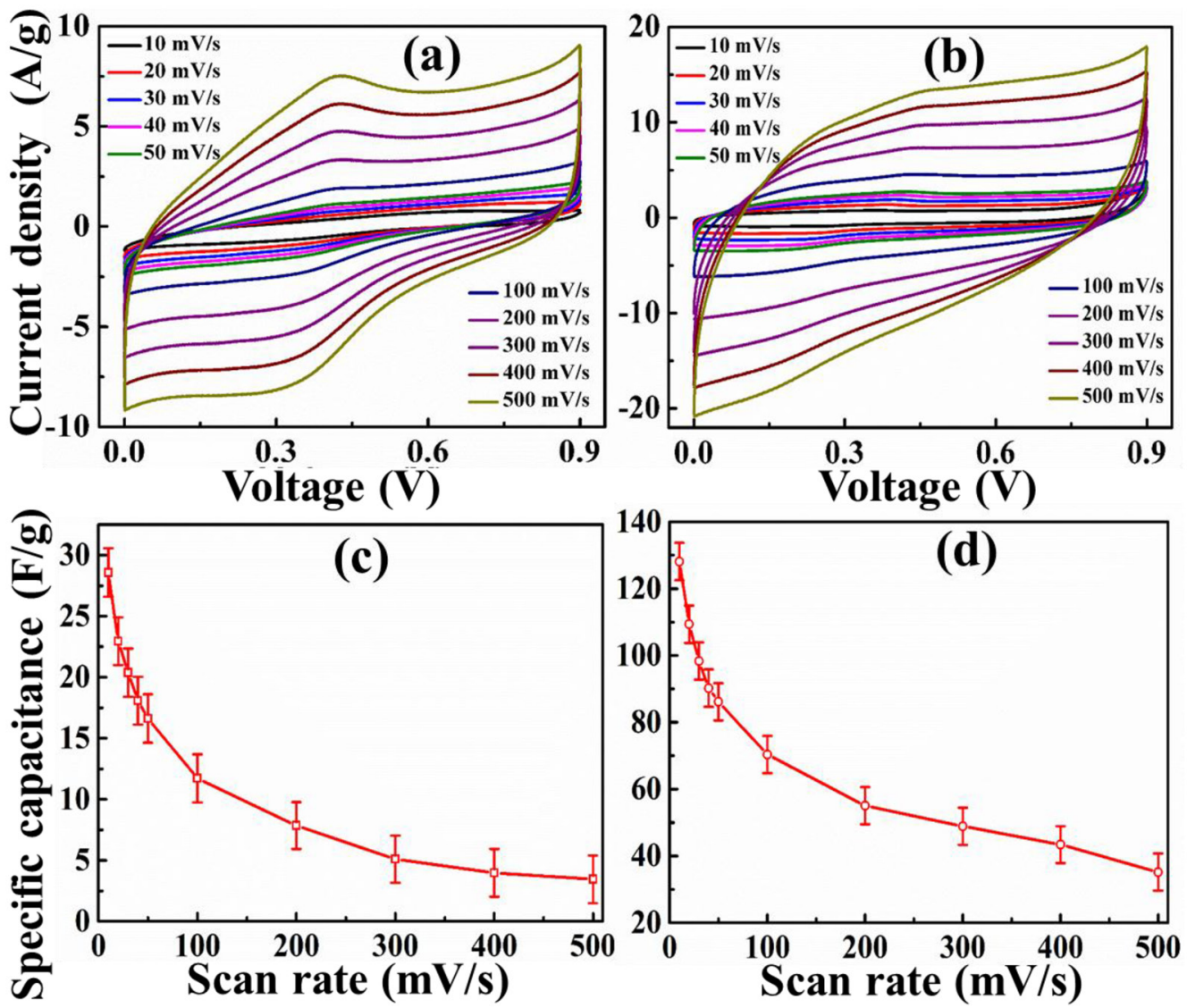

Figure 5. Electrochemical measurement of GO and Ru-rGO, CV curves of (a) GO (b) Ru-rGO at different scan rate; Variation of specific capacitance of with Scan rate of (c) GO and (d) Ru-rGO (error bars indicate \pm 1 S.D. from four measurements). 


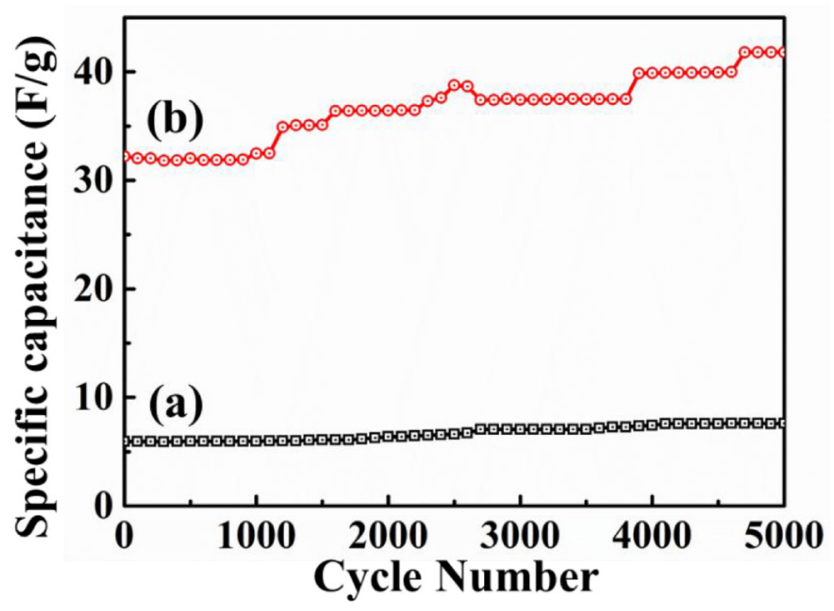

Figure 6. Cycling stability of (a) GO and (b) Ru-rGO measured at $500 \mathrm{mV} \mathrm{s}^{-1}$ for 5000 cycles.

Raman peaks in the range of 2575 to $2800 \mathrm{~cm}^{-1}$ have been deconvoluted using peak fitting software (Origin $\left.9{ }^{\circledR}\right)$ with Lorentzian functions and are shown in the inset of figure 4(b). The 2D band has four Lorentz components viz. $2 \mathrm{D}_{1 \mathrm{~A}}, 2 \mathrm{D}_{1 \mathrm{~B}}, 2 \mathrm{D}_{2 \mathrm{~A}}, 2 \mathrm{D}_{2 \mathrm{~B}}$ which are observed at $2611 \mathrm{~cm}^{-1}, 2646 \mathrm{~cm}^{-1}, 2668 \mathrm{~cm}^{-1}$, and $2720 \mathrm{~cm}^{-1}$ respectively [62]. This confirms the bilayer rGO formation with the implementation of bilayer graphene analogy [62]. The results are well in agreement with the number of layers estimated from XRD data. The average inter defect distance $\left(L_{\mathrm{D}}\right)$ and defect density $\left(n_{\mathrm{D}}\right)$ are estimated from the following equations (1) and (2) respectively.

$$
\begin{gathered}
L_{\mathrm{D}}^{2}=\frac{4.3 \times 10^{3}}{E_{\mathrm{L}}^{4}}\left[\frac{I_{\mathrm{D}}}{I_{\mathrm{G}}}\right]^{-1} \\
n_{\mathrm{D}}^{2}=\frac{1}{\pi L_{\mathrm{D}}^{2}}
\end{gathered}
$$

where, $E_{\mathrm{L}}=2.33 \mathrm{eV}$ is the laser excitation energy [56, 59]. The calculated $L_{\mathrm{D}}$ for $\mathrm{GO}$ is $13.1 \mathrm{~nm}$ and for $\mathrm{Ru}-$ rGO is $12.7 \mathrm{~nm}$. The defect density $\left(n_{\mathrm{D}}\right)$ for GO and Ru-rGO is found to be $18.5 \times 10^{6} \mathrm{~cm}^{-2}$ and $19.6 \times 10^{6}$ $\mathrm{cm}^{-2}$ respectively. The defect density for Ru-rGO is increased due to electron irradiation as compared to its counterpart GO. This increase is may be due to the binding of Ru nanoparticles as spacers into the rGO sheets.

The CV plots are determined from CV to understand the charge storage capacity of prepared samples. Figures 5(a) and (b) shows the CV plots of GO and $\mathrm{Ru}-\mathrm{rGO}$ at different scan rates in the range of 10 to 500 $\mathrm{mV} \mathrm{s}^{-1}$. The current density and area under the curve increases for both the samples with increasing scan rate. Figure 5(a) shows a distorted rectangular shaped $\mathrm{CV}$ curve with the presence of redox peaks at $0.3 \mathrm{~V}$ and $0.45 \mathrm{~V}$ representing the GO sample. In contrast, figure 5(b) shows a nearly rectangular shaped CV curve without the presence of the redox peaks in the Ru-rGO sample. This indicates the proper electric double layer capacitance (EDLC) behaviour with improved capaci- tive performance due to the improvement of charge transfer pathways in the electron irradiated sample. The volumetric current density for the applied potential window is higher in Ru-rGO as compared to its counterpart of GO indicating much higher utilization of electroactive species. The Ru-rGO samples have a higher volumetric current density than GO for all values of scan rates for the whole potential window. The specific capacitance of the GO and Ru-rGO is estimated from the CV curve using equation (3) [63].

$$
C_{\mathrm{S}}=\frac{1}{m v\left(V_{\mathrm{a}}-V_{\mathrm{c}}\right)} \int_{0}^{0.9} I(V) d V
$$

where $C_{\mathrm{S}}$ is specific capacitance $(\mathrm{F} / \mathrm{g}), \mathrm{m}$ is mass of active material, $v$ is scan rate $(\mathrm{mV} / \mathrm{s}), I$ is current, $\left(V_{\mathrm{a}}-V_{\mathrm{c}}\right)$ represents the potential window while $V_{\mathrm{a}}$ and $V_{c}$ are switching potentials. An area under CV curves in a given potential window is used to calculate specific capacitance. Figures 5(c) and (d) displays the specific capacitance as a function of scan rate. The specific capacitance of both Ru-rGO and GO decreased from $(128.1 \pm 5.59) \mathrm{F} \mathrm{g}^{-1}$ to $(35.15 \pm 5.52)$ $\mathrm{F} \mathrm{g}^{-1}$ and $(28.57 \pm 1.98) \mathrm{F} \mathrm{g}^{-1}$ to $(3.45 \pm 1.95) \mathrm{F} \mathrm{g}^{-1}$ respectively with an increase in scan rate from 10 to $500 \mathrm{mV} \mathrm{s}^{-1}$. This is due to the increase in diffusion components of current with an increase in scan rates.

Cycling stability of Ru-rGO is carried out using $\mathrm{CV}$ and shown in figure 6 . The specific capacitance retention up to 900 cycles is $\sim 99.4 \%$ for both GO and Ru-rGO while it increases to $130 \%$ at 5000 cycles for Ru-rGo sample. Ru-rGO has excellent cyclic stability revealing its application as an advanced electrode material for high-performance supercapacitors. Similar specific capacitance retention behavior is observed by Hassan et al [38]. The samples seem to follow the configuration of EDLC. In the case of EDLC, the charge storage process is nonfaradaic. On the basis of confirmation from TEM and FESEM, the $\mathrm{Ru}$ nanoparticles are present on the rGO sheets which also contribute to EDLC, con- 

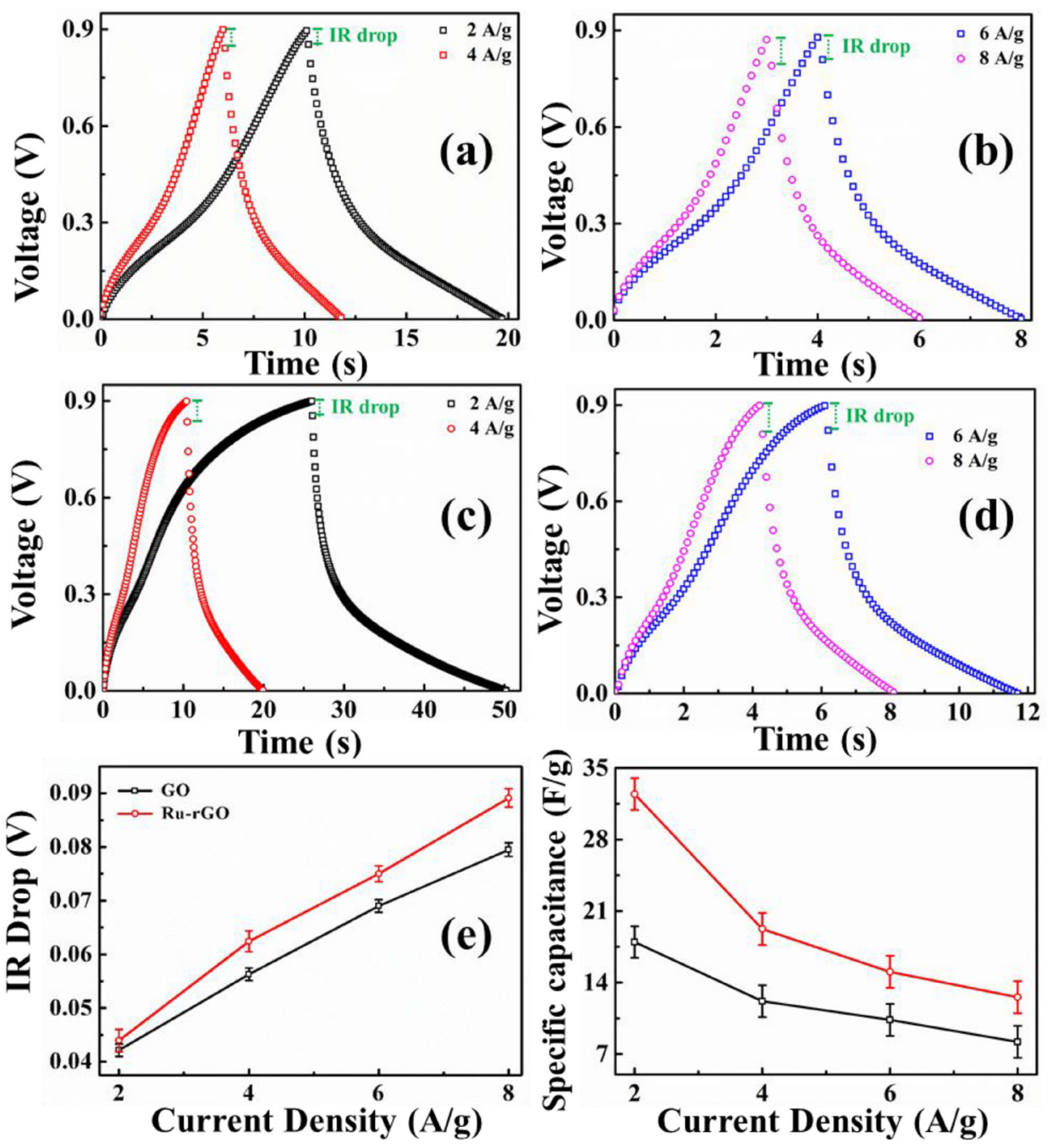

Figure 7. Galvanostatic charge/discharge curves of (a) GO (b) Ru-rGO at different current densities; variation of specific capacitance of (c) GO and (d) Ru-rGO at different current densities (error bars indicate \pm 1 S.D. from four measurements).

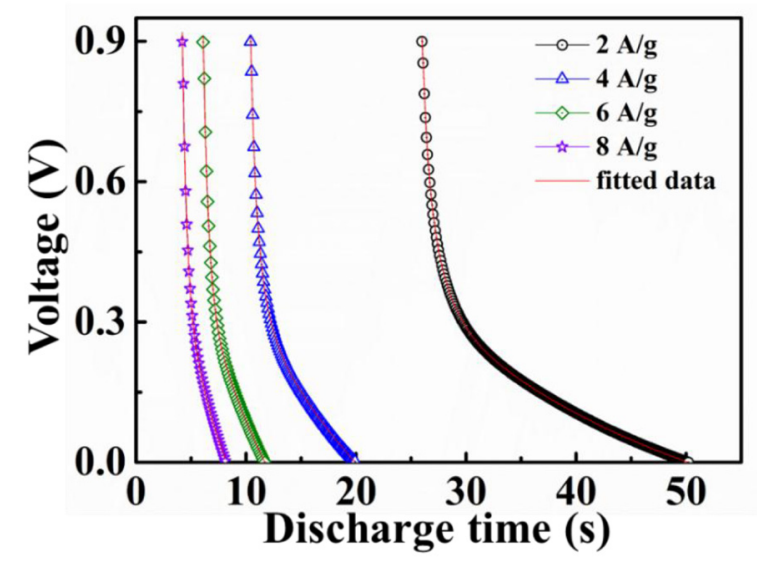

Figure 8. Exponential fitting of the discharge curve.

cluding that the energy storage mechanism is purely non-faradaic. Furthermore, as depicted from XRD and Raman analysis the synthesized Ru-rGO sample is composed of few layers with $\mathrm{Ru}$ nanoparticles as spacers and/or on the rGO sheet helps to increase the surface area with a reduction in the equivalent series resistance (ESR) and enhanced metallic conductivity, electrolyte penetration which promotes fast ion exchange.

The galvanostatic charge/discharge curves of the $\mathrm{GO}$ and $\mathrm{Ru}-\mathrm{rGO}$ at different current densities ranging from 2 to $8 \mathrm{~A} \mathrm{~g}^{-1}$ are presented in figures 7 (a)-(d). 
Table 2. Fitting parameters of the discharge curve.

\begin{tabular}{|c|c|c|c|c|c|c|}
\hline \multirow{2}{*}{$\begin{array}{l}\text { Current density } \\
\left(\mathrm{A} \mathrm{g}^{-1}\right)\end{array}$} & \multicolumn{6}{|c|}{ Fitting parameters } \\
\hline & $A_{1}$ & $t_{1}$ & $A_{2}$ & $t_{2}$ & $A_{3}$ & $t_{3}$ \\
\hline 2 & 0.38691 & 1.68345 & 0.55675 & 25.51159 & 0.39923 & 0.48802 \\
\hline 4 & 0.27517 & 1.05203 & 0.78162 & 17.42795 & 0.32631 & 0.44669 \\
\hline 6 & 0.42424 & 10.26151 & 0.41633 & 10.26144 & 0.48386 & 0.47538 \\
\hline 8 & 0.33214 & 4.86532 & 0.34128 & 4.86532 & 0.34128 & 0.47538 \\
\hline
\end{tabular}

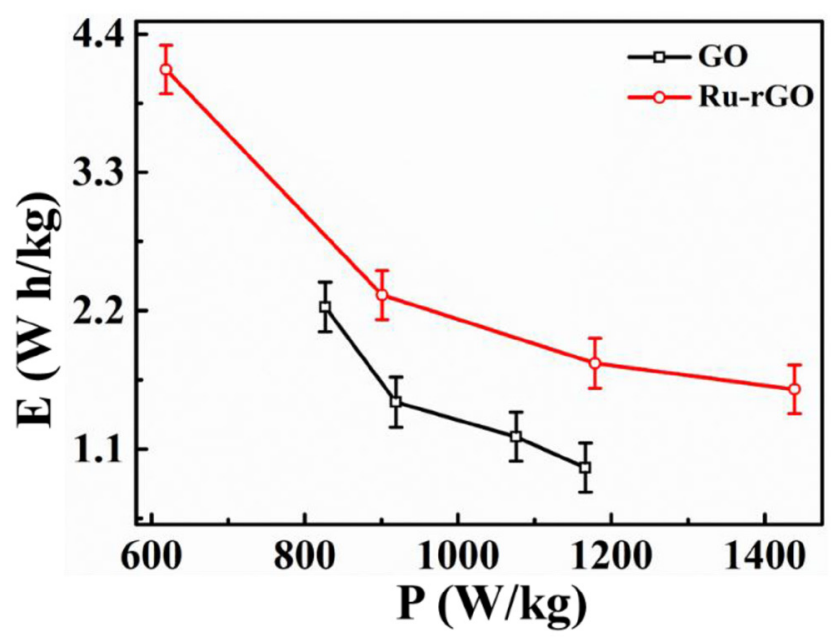

Figure 9. Ragone plot for GO (black) and Ru-rGO (red) (error bars indicate \pm 1 S.D. from four measurements).

Figures $7(\mathrm{a})$ and (b) shows a symmetric charging/ discharging phenomenon in GO for all applied current densities. The nature of the charging and discharging curves of Ru-rGO (figures 7 (a) and (b)) do not follow the triangular shape as in the GO sample. The Ru-rGO sample shows a longer discharge time than GO indicating higher specific capacitance may be due to the formation of few-layered Ru-rGO. The nature of the charge/discharge curve is attributed to the charge re-distribution phenomenon in the porous electrode [64]. It is seen that the discharge curve exhibits the slope, which may be due to the IRdrop. The dependence of IR drop on applied current density is shown in figure 7(e). From the plot, it is seen that IR drop increases with increase in applied current density for both GO and Ru-rGO. Ru-rGO exhibits a slightly higher IR drop as compared to GO indicating Ru-rGO exhibits relatively large internal resistance. Charge storage in Ru-rGO sheets takes place through adsorption/desorption of ions and/or electrons. The edge defects, oxygen-containing groups and active sites present on rGO provide a huge interface for the same [65]. From the charge/discharge curve for $\mathrm{Ru}$ rGO it is seen that, below $0.2 \mathrm{~V}$ a very slow discharge takes place (at $2 \mathrm{~A} \mathrm{~g}^{-1}$ applied current density). This may be due to the slower release of absorbed charges on the surface of Ru-rGO. Furthermore, the particleon-sheet structure of Ru-rGO nanocomposite can increase the number of electroactive sites and accelerate the diffusion of ions by providing low-resistance pathways [28].
The specific capacitance of the GO and Ru-rGO is estimated from the galvanostatic charge/discharge curves using equation (4) [20].

$$
C_{S}=\frac{I \Delta t}{m \Delta V}
$$

Where $I / m$ is the constant current density for discharge, $\Delta t$ is the time of discharge, $\Delta V$ is the potential window for discharge. The specific capacitance values calculated from the charge/discharge curve are found to be $(17.97 \pm 1.54) \mathrm{F} \mathrm{g}^{-1}$ and $(32.45 \pm 1.55)$ $\mathrm{F} \mathrm{g}^{-1}$ at $2 \mathrm{~A} \mathrm{~g}^{-1}$ which drops to $(8.2 \pm 1.55) \mathrm{F} \mathrm{g}^{-1}$ and $(12.57 \pm 1.56) \mathrm{F} \mathrm{g}^{-1}$ at a current density of $8 \mathrm{~A}$ $\mathrm{g}^{-1}$ for GO and Ru-rGO respectively. It is seen that a rapid decrease in specific capacitance occurs at higher current density. Finally, it can be inferred that Ru-rGO forms a unique structure with good stability and integrity. Furthermore, the Ru nanoparticles act as a spacer that prevents the rGO layers from agglomeration [27, 28, 37].

The discharging curve is fitted with the exponential function $y=y_{0}+A_{1} \times \exp \left(-\frac{x-x_{0}}{t_{1}}\right)+$ $A_{2} \times \exp \left(-\frac{x-x_{0}}{t_{2}}\right)+A_{3} \times \exp \left(-\frac{x-x_{0}}{t_{3}}\right)$ as shown in figure 8 . The nature of the discharge curve implies that the discharging process involves a three current decay process [66], in which first discharge time $\left(t_{1}\right)$ is associated to the IR drop (Activation polarization) which occurs due to the instant (charge release from electrode/electrolyte) discharge from electrolyte recombination. The second discharging time $\left(t_{2}\right)$ is related to 
ohmic polarization which arises from the resistance of the electrolyte, the materials of construction of the electrodes and the conductive diluent, current collectors, and contact between particles of the active mass and conductive diluent or from a resistive film on the surface of the electrode. The last one discharging time $\left(t_{3}\right)$ is associated with concentration polarization arising from partial mass transport abilities like limited diffusion of dynamic species to and from the electrode surface. The fitting parameters of the discharge curve are listed in table 2 .

The energy density and power density are evaluated using equation (5) [38],

$$
E=\frac{\frac{1}{2} C_{\mathrm{s}} \Delta V^{2}}{3.6} \text { and } P=\frac{E}{t_{\mathrm{d}}} \times 3600,
$$

where $C_{\mathrm{S}}$ is the specific capacitance in $\mathrm{F}^{-1}, m$ is the mass of electrode material in grams, $I$ is the current in ampere, and $t_{\mathrm{d}}$ is the discharge time in seconds. IR drop is excluded from the potential value during measurement of power density and energy density. The values 3.6 and 3600 in the energy density and power density equation are the result of unit conversions of mass and time from $\mathrm{g}$ to $\mathrm{kg}$ and from $\mathrm{sec}$ to hour, respectively. Figure 9 shows the Ragone plot for GO and Ru-rGO. GO has highest energy density $(2.23 \pm 0.19) \mathrm{W} \mathrm{h} \mathrm{kg}^{-1}$ at a power density of 826.53 $\mathrm{W} \mathrm{k}^{-1} \mathrm{~g}$ while $\mathrm{Ru}-\mathrm{rGO}$ possesses the highest energy density of ( $4.11 \pm 0.19) \mathrm{W} \mathrm{h} \mathrm{kg}^{-1}$ at a power density of $618.75 \mathrm{~W} \mathrm{~kg}^{-1}$. The energy density decreases for both the samples at higher power density due to fast voltage decay at higher values of power.

\section{Conclusion}

We have developed few layer Ru-rGO by an in situ 6 $\mathrm{MeV}$ electron beam induced reduction method. This method shows an efficient reduction of GO to rGO without the use of reducing or stabilizing agent. GO and Ru-rGO based supercapacitors exhibit the highest specific capacitance of $(28.57 \pm 1.98) \mathrm{F} \mathrm{g}^{-1}$ and $(128.1 \pm 5.59) \mathrm{Fg}^{-1}$ at $10 \mathrm{mV} \mathrm{s}^{-1}$ scan rate respectively. The shape of the voltammogram is retained up to a high scan rate of $100 \mathrm{mV} \mathrm{s}^{-1}$ which indicates that Ru-rGO has an excellent rate performance for the supercapacitor device. Among both supercapacitors, Ru-rGO based supercapacitor shows appreciably high specific capacitance, with a capacity retention of about $99.4 \%$ up to 900 cycles which further increases to $130 \%$ at 5000 cycles for Ru-rGO. The highest energy density of $(4.11 \pm 0.19) \mathrm{W} \mathrm{h} \mathrm{kg}^{-1}$ and power density of 1.44 $\mathrm{kW} \mathrm{kg}^{-1}$ are achieved with Ru-rGO supercapacitor.

\section{Acknowledgment}

The authors acknowledge BRNS (Research project Number 34/14/05/2015/BRNS), Government of India for financial support. Gratitude is due to Dr Lesley Hanna of the Institute of Materials and
Manufacturing, Brunel University London for help in preparing the manuscript.

\section{ORCID iDs}

Nilesh G Saykar (1) https://orcid.org/0000-0001-77072190

V N Bhoraskar (1) https://orcid.org/0000-0001-73002136

Asim K Ray @ https://orcid.org/0000-0002-5392-0041 S K Mahapatra (1) https://orcid.org/0000-0001-73703995

\section{References}

[1] Arya A and Sharma A L 2017 Insights into the use of polyethylene oxide in energy storage/conversion devices: a critical review J. Phys. D: Appl. Phys. 50443002

[2] Saykar N G, Pilania R K, Banerjee I and Mahapatra S K 2018 Synthesis of $\mathrm{NiO}-\mathrm{Co}_{3} \mathrm{O}_{4}$ nanosheet and its temperaturedependent supercapacitive behavior J. Phys. D: Appl. Phys. 51475501

[3] Pritam, Arya A and Sharma A L 2019 Dielectric relaxations and transport properties parameter analysis of novel blended solid polymer electrolyte for sodium-ion rechargeable batteries $J$. Mater. Sci. 54 7131-55

[4] Stauss S and Honma I 2018 Biocompatible batteriesmaterials and chemistry, fabrication, applications, and future prospects Bull. Chem. Soc. Japan 91 492-505

[5] Arya A and Sharma A L 2019 Electrolyte for energy storage/ conversion $\left(\mathrm{Li}^{+}, \mathrm{Na}^{+}, \mathrm{Mg}^{2+}\right)$ devices based on PVC and their associated polymer: a comprehensive review J. Solid State Electrochem. 23 997-1059

[6] Xue Y, Zhang Q, Wang W, Cao H, Yang Q and Fu L 2017 Opening two-dimensional materials for energy conversion and storage: a concept Adv. Energy Mater. 71602684

[7] Wu Z, Li L, Yan J M and Zhang X B 2017 Materials design and system construction for conventional and new-concept supercapacitors Adv. Sci. 41600382

[8] Khan A H, Ghosh S, Pradhan B, Dalui A, Shrestha L K, Acharya S and Ariga K 2017 Two-dimensional (2D) nanomaterials towards electrochemical nanoarchitectonics in energy-related applications Bull. Chem. Soc. Japan 90 627-48

[9] Kumar K S, Choudhary N, Jung Y and Thomas J 2018 Recent advances in two-dimensional nanomaterials for supercapacitor electrode applications ACS Energy Lett. 3482-95

[10] Yun Q, Lu Q, Zhang X, Tan C and Zhang H 2018 Threedimensional architectures constructed from transition-metal dichalcogenide nanomaterials for electrochemical energy storage and conversion Angew. Chem., Int. Ed. Engl. 57 626-46

[11] Li F and Zhou Z 2018 Micro/nanostructured materials for sodium ion batteries and capacitors Small 141702961

[12] Yi H, Jiajie L and Yongsheng C 2012 An overview of the applications of graphene-based materials in supercapacitors Small 8 1805-34

[13] Brownson D A, Kampouris D K and Banks C E 2011 An overview of graphene in energy production and storage applications J. Power Sources 196 4873-85

[14] Zhu J, Yang D, Yin Z, Yan Q and Zhang H 2014 Graphene and graphene-based materials for energy storage applications Small 10 3480-98

[15] Xia J, Chen F, Li J and Tao N 2009 Measurement of the quantum capacitance of graphene Nat. Nanotechnol. 4505

[16] Bo Z, Shuai X, Mao S, Yang H, Qian J, Chen J and Cen K 2014 Green preparation of reduced graphene oxide for sensing and energy storage applications Sci. Rep. 44684

[17] Deng W, Ji X, Chen Q and Banks C E 2011 Electrochemical capacitors utilising transition metal oxides: an update of recent developments RSC Adv. 11171-8 
[18] Cao X, Shi Y, Shi W, Lu G, Huang X, Yan Q and Zhang H 2011 Preparation of novel 3D graphene networks for supercapacitor applications Small 7 3163-8

[19] Yan J, Wei T, Shao B, Ma F, Fan Z, Zhang M and Wei F 2010 Electrochemical properties of graphene nanosheet/carbon black composites as electrodes for supercapacitors Carbon 48 1731-7

[20] Wang C, Li D, Too C O and Wallace G G 2009 Electrochemical properties of graphene paper electrodes used in lithium batteries Chem. Mater. 21 2604-6

[21] Stankovich S, Dikin D A, Piner R D, Kohlhaas KA, Kleinhammes A, Jia Y and Ruoff R S 2007 Synthesis of graphene-based nanosheets via chemical reduction of exfoliated graphite oxide Carbon 45 1558-65

[22] Yang X, Zhu J, Qiu L and Li D 2011 Bioinspired effective prevention of restacking in multilayered graphene films: towards the next generation of high-performance supercapacitors Adv Mater. 23 2833-8

[23] Liu C, Yu Z, Neff D, Zhamu A and Jang B Z 2010 Graphenebased supercapacitor with an ultrahigh energy density Nano Lett. $104863-8$

[24] Weng Z, Su Y, Wang D W, Li F, Du J and Cheng H M 2011 Graphene-cellulose paper flexible supercapacitors Adv. Energy Mater. 1917-22

[25] Hu C C, Wang C-W, Chang K-H and Chen M-G 2015 Anodic composite deposition of $\mathrm{RuO}_{2} /$ reduced graphene oxide/carbon nanotube for advanced supercapacitors Nanotechnology 26274004

[26] Lou B-S, Veerakumar P, Chen S-M, Veeramani V, Madhu R and Liu S-B 2016 Ruthenium nanoparticles decorated curl-like porous carbons for high performance supercapacitors Sci. Rep. 619949

[27] Kim J-Y, Kim K-H, Yoon S-B, Kim H-K, Park S-H and Kim K-B 2013 In situ chemical synthesis of ruthenium oxide/reduced graphene oxide nanocomposites for electrochemical capacitor applications Nanoscale 5 6804-11

[28] Zheng L, Zhang G, Zhang M, Guo S and Liu Z H 2012 Preparation and capacitance performance of Ag-graphene based nanocomposite J. Power Sources 201 376-81

[29] Amir F, Pham V and Dickerson J 2015 Facile synthesis of ultra-small ruthenium oxide nanoparticles anchored on reduced graphene oxide nanosheets for high-performance supercapacitors RSCAdv. 567638-45

[30] Huang X, Qi X, Boey F and Zhang H 2012 Graphene-based composites Chem. Soc. Rev. 41 666-86

[31] Liu S, Yan J, He G, Zhong D, Chen J, Shi L and Jiang H 2012 Layer-by-layer assembled multilayer films of reduced graphene oxide/gold nanoparticles for the electrochemical detection of dopamine J. Electroanal. Chem. 672 40-4

[32] Iliut M, Leordean C, Canpean V, Teodorescu C-M and Astilean S 2013 A new green, ascorbic acid-assisted method for versatile synthesis of Au-graphene hybrids as efficient surface-enhanced Raman scattering platforms J. Mater. Chem. C 1 4094-104

[33] Ali M A, Singh C, Srivastava S, Admane P, Agrawal V V, Sumana G and Malhotra B D 2017 Graphene oxide-metal nanocomposites for cancer biomarker detection RSCAdv. 7 35982-91

[34] Yang B, Bin D, Wang H, Zhu M, Yang P and Du Y 2015 High quality Pt-graphene nanocomposites for efficient electrocatalytic nitrite sensing Colloids Surf. A 481 43-50

[35] Xie G, Forslund M and Pan J 2014 Direct electrochemical synthesis of reduced graphene oxide (rGO)/copper composite films and their electrical/electroactive properties ACS Appl. Mater. Interfaces 6 7444-55

[36] Shayeh J S, Ehsani A, Ganjali M R, Norouzi P and Jaleh B 2015 Conductive polymer/reduced graphene oxide/Au nano particles as efficient composite materials in electrochemical supercapacitors Appl. Surf. Sci. 353 594-9

[37] Si Y and Samulski E T 2008 Exfoliated graphene separated by platinum nanoparticles Chem. Mater. 20 6792-7

[38] Hassan H K, Atta N F, Hamed M M, Galal A and Jacob T 2017 Ruthenium nanoparticles-modified reduced graphene prepared by a green method for high-performance supercapacitor application in neutral electrolyte RSC Adv. $711286-96$

[39] Yan Z, Ma L, Zhu Y, Lahiri I, Hahm M G, Liu Z and Sun Z 2012 Three-dimensional metal-graphene-nanotube multifunctional hybrid materials ACS Nano 7 58-64

[40] Hareesh K, Joshi R, Dahiwale S, Bhoraskar V and Dhole S 2016 $6 \mathrm{MeV}$ energy electron beam assisted synthesis of Ag-rGO nanocomposite and its photocatalytic activity Mater Lett. 16435-8

[41] Yang Y, Chen L, Li D-Y, Yi R-B, Mo J-W, Wu M-H and Xu G 2019 Controllable reduction of graphene oxide by electronbeam irradiation RSCAdv. 93597-604

[42] Yu Z, Sun S and Huang M 2016 Electrodeposition of gold nanoparticles on electrochemically reduced graphene oxide for high performance supercapacitor electrode materials Int. J. Electrochem. Sci. $113643-50$

[43] Chaudhary M, Doong R-A, Kumar N and Tseng TY 2017 Ternary Au/ZnO/rGO nanocomposites electrodes for high performance electrochemical storage devices Appl. Surf. Sci. $420118-28$

[44] Das H T, Mahendraprabhu K, Maiyalagan T and Elumalai P 2017 Performance of solid-state hybrid energy-storage device using reduced graphene-oxide anchored sol-gel derived $\mathrm{Ni}$ / NiO nanocomposite Sci. Rep. 715342

[45] Kamila S, Mohanty B, Samantara A K, Guha P, Ghosh A, Jena B and Jena B K 2017 Highly active 2D layered $\mathrm{MoS}_{2}$ rGO hybrids for energy conversion and storage applications Sci. Rep. 78378

[46] Saraf M, Natarajan K and Mobin S M 2018 Emerging robust heterostructure of $\mathrm{MoS}_{2}$-rGO for high-performance supercapacitors ACS Appl. Mater. Interfaces 10 16588-95

[47] Kirubasankar B, Vijayan S and Angaiah S 2019 Sonochemical synthesis of a 2D-2D MoSe 2 /graphene nanohybrid electrode material for asymmetric supercapacitors Sustain. Energy Fuel 3467-77

[48] $\mathrm{Hu} \mathrm{R}$, Zhao J and Zheng J 2017 Synthesis of $\mathrm{SnO}_{2} / \mathrm{rGO}$ hybrid materials by sol-gel/thermal reduction method and its application in electrochemical capacitors Mater Lett. 197 59-62

[49] Navarro-Suárez A M, Maleski K, Makaryan T, Yan J, Anasori B and Gogotsi Y 2018 2D titanium carbide/reduced graphene oxide heterostructures for supercapacitor applications Batteries Supercaps 133-8

[50] Wang X, Lin S, Tong H, Huang Y, Tong P, Zhao B and Zhu X 2019 Two-dimensional V4C3 MXene as high performance electrode materials for supercapacitors Electrochim. Acta 307 414-21

[51] Belloni J 2006 Nucleation, growth and properties of nanoclusters studied by radiation chemistry: application to catalysis Catal. Today 113 141-56

[52] Buxton G V, Greenstock C L, Helman W P and Ross A B 1988 Critical review of rate constants for reactions of hydrated electrons, hydrogen atoms and hydroxyl radicals (.OH/.O - in aqueous solution J. Phys. Chem. Ref. Data 17 513-886

[53] Flyunt R, Knolle W, Kahnt A, Prager A, Lotnyk A, Malig J and Abel B 2014 Mechanistic aspects of the radiation-chemical reduction of graphene oxide to graphene-like materials Int. J. Radiat. Biol. 90 486-94

[54] Ding Y, Zhang P, Zhuo Q, Ren H, Yang Z and Jiang Y 2011 A green approach to the synthesis of reduced graphene oxide nanosheets under UV irradiation Nanotechnology 22215601

[55] Wei J, Zhang X, Qiu J and Weeks B L 2015 Thermal kinetics and thermo-mechanical properties of graphene integrated fluoroelastomer J. Polym. Sci. B 53 1691-700

[56] Banerjee I, Faris T, Stoeva Z, Harris P G, Chen J, Sharma A K and Ray A K 2016 Graphene films printable on flexible substrates for sensor applications 2D Mater. 4015036

[57] Ferrari A C 2007 Raman spectroscopy of graphene and graphite: disorder, electron-phonon coupling, doping and nonadiabatic effects Solid State Commun. 143 47-57

[58] Malard L, Pimenta M, Dresselhaus G and Dresselhaus M 2009 Raman spectroscopy in graphene Phys. Rep. 473 51-87 
[59] Ferrari A C and Basko D M 2013 Raman spectroscopy as a versatile tool for studying the properties of graphene $\mathrm{Nat}$. Nanotechnol. 8235

[60] Chan HY H, Takoudis C G and Weaver M J 1997 High-pressure oxidation of ruthenium as probed by surface-enhanced Raman and x-ray photoelectron spectroscopies J. Catal. 172 336-45

[61] Cançado L G, Jorio A, Ferreira E M, Stavale F, Achete C, Capaz R and Ferrari A 2011 Quantifying defects in graphene via Raman spectroscopy at different excitation energies Nano Lett. 113190-6

[62] Ferrari A C, Meyer J, Scardaci V, Casiraghi C, Lazzeri M, Mauri F and Roth S 2006 Raman spectrum of graphene and graphene layers Phys. Rev. Lett. 97187401
[63] Islam M, Chidembo A T, Aboutalebi S H, Cardillo D, Liu H K, Konstantinov K and Dou S X 2014 Liquid crystalline graphene oxide/PEDOT: PSS self-assembled 3D architecture for binder-free supercapacitor electrodes Frontiers Energy Res. 231

[64] Suraj S, Mejo A, Muralidharan M and Ansari S 2018 Self discharge and voltage recovery in graphene supercapacitors IEEE Trans. Power Electr. 33 10410-8

[65] Li F, Jiang X, Zhao J and Zhang S 2015 Graphene oxide: a promising nanomaterial for energy and environmental applications Nano Energy 16 488-515

[66] Winter M and Brodd R J 2004 What are batteries, fuel cells, and supercapacitors? Chem. Rev. 104 4245-69 